\title{
Lymphovascular invasion, race, and the 21-gene recurrence score in early estrogen receptor-positive breast cancer
}

\author{
Della Makower $\mathbb{D}^{1 凶}$, Juan $\operatorname{Lin}^{2}$, Xiaonan Xue ${ }^{2}$ and Joseph A. Sparano (iD
}

Lymphovascular invasion (LVI) and Black race are associated with poorer prognosis in early breast cancer (EBC). We evaluated the association between $L V I$ and race, and whether LVI adds prognostic benefit to the 21-gene recurrence score (RS) in EBC. Women with ER+ HER2 - EBC measuring up to $5 \mathrm{~cm}$, with 0-3 involved axillary nodes, diagnosed between 1 January 2010 and 1 January 2014, who underwent surgery as first treatment and had available RS, were identified in the NCDB database. Bivariate associations between two categorical variables were examined using chi-square test. Multivariate Cox proportional hazards model were used to assess the association of $\mathrm{LVI}$, race, and other covariates with overall survival (OS). 77,425 women, 65,018 node-negative (N0), and 12,407 with $1-3$ positive $(\mathrm{N}+$ ) nodes, were included. $\mathrm{LVI}$ was present in $12.7 \%$, and associated with poor grade, $\mathrm{RS} 26-100$, and $\mathrm{N}+$ (all $p<0.0001$ ), but not Black race. In multivariate analysis, LVI was associated with worse OS in NO [HR 1.37 (95\% Cl 1.27, 1.57], but not N+ EBC. LVI was associated with worse OS in N0 patients with RS 11-25 [HR $1.31(95 \% \mathrm{Cl} 1.09,1.57)]$ and $\geq 26$ [HR $1.58(95 \% \mathrm{Cl}$ $1.30,1.93)$ ], but not RS $0-10$. No interaction between LVI and chemotherapy benefit was seen. Black race was associated with worse OS in NO (HR 1.21, $p=0.009$ ) and N+ (HR 1.37, $p=0.015)$ disease. LVI adds prognostic information in ER+, HER2 -, N0 BCA with RS 11-100, but does not predict chemotherapy benefit. Black race is associated with worse OS, but not LVI.

npj Breast Cancer (2021)7:20; https://doi.org/10.1038/s41523-021-00231-x

\section{INTRODUCTION}

Lymphovascular invasion (LVI), the presence of tumor cells within the lumen of lymphatic or vascular system in the tumor primary site, is associated with a higher risk of regional lymph node metastasis at diagnosis and subsequent distant recurrence in early breast cancer $(\mathrm{EBC})^{1-7}$. Multiparameter gene expression assays also provide prognostic information for distant recurrence in hormone receptor-positive (HR+), HER2 negative breast (HER2-) cancer ${ }^{8,9}$. The 21 -gene recurrence score (RS) assay is both prognostic for recurrence and predictive of chemotherapy benefit in $\mathrm{EBC}^{10-13}$, including women with up to three positive axillary nodes $^{14,15}$. The extent to which LVI adds prognostic information or predictive information for chemotherapy benefit to the 21-gene RS or other assays has not been adequately evaluated ${ }^{16}$.

Black race has also been associated with worse prognosis in EBC. Although disparities in access to care play a role in Black patients' poorer outcomes ${ }^{17,18}$, survival gaps are also seen for Black women receiving contemporary adjuvant therapy on $\mathrm{NCl}$ sponsored clinical trials ${ }^{19-22}$, suggesting that factors other than care disparities contribute to their poorer prognosis. For example, in the TAILORx trial, Black race was associated with a 1.4-fold higher risk of recurrence after adjustment for clinicopathologic characteristics, despite similar chemotherapy and endocrine therapy use and similar RS distribution ${ }^{23}$. Thus, biological factors that are not captured by the 21-gene RS, including racial differences in the tumor microenvironment ${ }^{24,25}$, may contribute to the worse outcomes for Black women with EBC.

The presence of $L V I$ represents a manifestation of an interaction between the tumor and its microenvironment. The molecular mechanisms underlying LVI are complex and not fully defined, but pathways involving cytokine-receptor interactions have been implicated $^{26-28}$. Studies evaluating racial differences in BCA tumor microenvironment have described the differential expression of genes associated with inflammation and angiogenesis ${ }^{24,25,29-31}$, as well as increased microvessel density ${ }^{29,31}$ and increased numbers of tumor-associated macrophages ${ }^{29,32}$, which correlate with increased lymphangiogenesis and $\mathrm{LVI}^{33-35}$, in tumors belonging to Black women. Although no studies have directly compared racial differences in LVI in BCA, a multinational study evaluating clinicopathologic characteristics of urothelial tumors in white and Asian patients showed a significant racial difference in the rate of $\mathrm{LVI}^{36}$. No prior studies have evaluated the impact of LVI, RS, and race in the same population. We, therefore, sought to assess whether LVI adds prognostic or predictive information to the 21gene RS, and whether there is an association between LVI and race that may contribute to racial disparities in EBC.

\section{RESULTS}

\section{Patient characteristics}

As shown in Fig. 1, of 2,696,734 women with breast cancer in the National Cancer Database (NCDB), 77,425 met inclusion criteria for this analysis, 65,018 of whom had no nodal involvement (N0), and 12,407 who had involvement of 1-3 axillary nodes $(\mathrm{N}+)$. Clinicopathologic and demographic characteristics of the study population are shown in Table 1. In all, 67,998 (87.8\%) of patients were white, 5750 (7.4\%) were Black, 3096 (4.0\%) were members of other racial groups, and $581(0.8 \%)$ had no racial information available. LVI was reported as present in 9856 (12.7\%), absent in 59,305 (76.60\%), and unknown in 8264 (10.67\%).

Association between LVI and other clinicopathologic features As shown in Table 2, LVI was significantly associated with other poor prognostic clinicopathologic features, including axillary node metastasis (37.0\% vs $12.2 \%)$, tumor size larger than $2 \mathrm{~cm}(37.1 \%$ vs. $21.0 \%)$, grade 3 histology ( $28.0 \%$ vs. $13.6 \%)$, and high RS of $26-100$

\footnotetext{
${ }^{1}$ Montefiore Einstein Center for Cancer Care, Bronx, NY, USA. ${ }^{2}$ Albert Einstein Cancer Center, Bronx, NY, USA. ${ }^{凶}$ email: dmakower@montefiore.org
} 


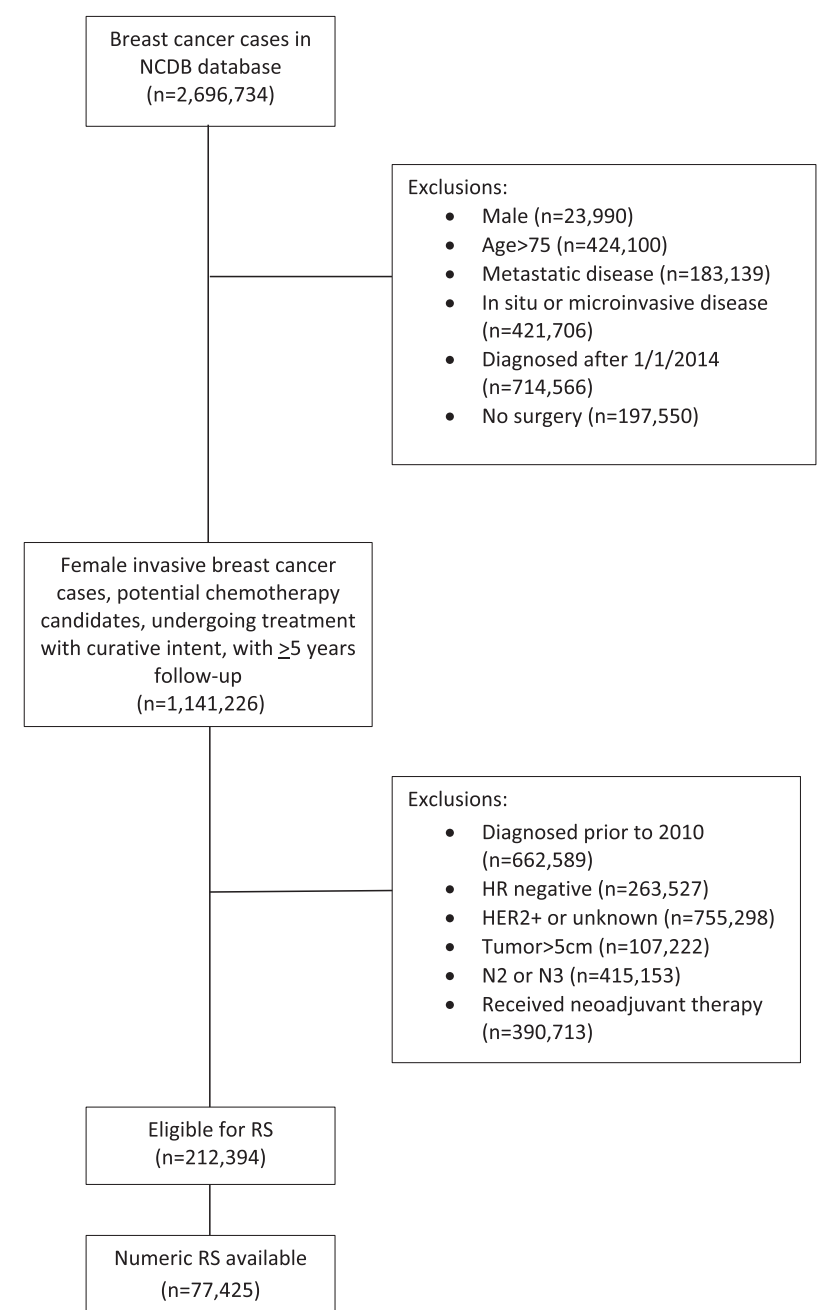

Fig. 1 CONSORT flow diagram for patient selection. NCDB National Cancer Database, HR hormone receptor, N2, 4-9 involved axillary nodes, N3 10 or more involved axillary nodes, RS 21-gene recurrence score.

( $21.5 \%$ vs. $13.9 \%)$. These differences were substantial in magnitude and clinically relevant.

\section{Associations between race and other clinicopathologic features}

Also shown in Table 2, Black race was associated with higher rates of axillary node metastasis (17.2\% for Black women vs. $16.0 \%$ for white women vs. $15.3 \%$ for women of other races), tumor size larger than $2 \mathrm{~cm}(27.1 \%$ vs. $22.8 \%$ vs. $25.4 \%)$, grade 3 histology ( $21.8 \%$ vs. $15.0 \%$ vs. $17.6 \%)$, and high RS of $26-100(20.4 \%$ vs. $14.5 \%$ vs. $16.3 \%$ ). Although these differences were statistically significant, the absolute differences were marginal or modest. In contrast, LVI was slightly lower in Black women than in white women or women of other races $(12.3 \%$ vs. $12.8 \%$ vs $13.2 \%, p=$ 0.0365).

\section{Association between $\mathrm{LVI}$, race, and OS}

Clinicopathologic features associated with poorer overall survival (OS) in univariate analysis were axillary nodal involvement, larger tumor size, higher grade, increasing RS, presence of $\mathrm{LVI}$, and increased number of comorbidities ( $p<0.0001$ for all). Demographic factors associated with poorer OS in univariate analysis were Black race, older age, lower median educational level, and lower median income ( $p<0.0001$ for all) (Supplemental Table 1).
Table 1. Patient characteristics $(n=77,425)$.

\begin{tabular}{|c|c|}
\hline Characteristic & Number (\%) \\
\hline Age (median, IQR) & $59(50-65)$ \\
\hline \multicolumn{2}{|l|}{ Race } \\
\hline White & $67998(87.8 \%)$ \\
\hline Black & $5750(7.4 \%)$ \\
\hline Other & $3096(4.0 \%)$ \\
\hline Unknown & $581(0.8 \%)$ \\
\hline \multicolumn{2}{|l|}{ LVI } \\
\hline Present & $9856(12.7 \%)$ \\
\hline Absent & $59,305(76.6 \%)$ \\
\hline Unknown & $8264(10.7 \%)$ \\
\hline \multicolumn{2}{|l|}{ Tumor size $(\mathrm{mm})$} \\
\hline $0-20$ & $59,396(76.7 \%)$ \\
\hline $21-50$ & $18,029(23.3 \%)$ \\
\hline \multicolumn{2}{|c|}{ Lymph node involvement } \\
\hline 0 & $65,018(84.0 \%)$ \\
\hline $1-3^{a}$ & $12,407(16.0 \%)$ \\
\hline \multicolumn{2}{|l|}{ Grade } \\
\hline 1 & 21,069 (27.2\%) \\
\hline 2 & $40,188(51.9 \%)$ \\
\hline 3 & $12,077(15.6 \%)$ \\
\hline Unknown & 4091 (5.3\%) \\
\hline \multicolumn{2}{|l|}{ Recurrence score } \\
\hline $0-10$ & $17,390(22.5 \%)$ \\
\hline $11-25$ & $48,398(62.5 \%)$ \\
\hline $26-100$ & $11,637(15.0 \%)$ \\
\hline \multicolumn{2}{|c|}{ Charlson-Deyo comorbidities } \\
\hline 0 & $65,906(85.1 \%)$ \\
\hline 1 & $9761(12.6 \%)$ \\
\hline 2 & $1433(1.8 \%)$ \\
\hline 3 or more & $325(0.4 \%)$ \\
\hline \multicolumn{2}{|c|}{ Median income quartile } \\
\hline$<\$ 38,000$ & $9001(11.6 \%)$ \\
\hline$\$ 38,000-\$ 47,999$ & $15,080(19.5 \%)$ \\
\hline$\$ 48,000-\$ 62,999$ & $21,028(27.2 \%)$ \\
\hline$>\$ 63,000$ & $32,146(41.6 \%)$ \\
\hline \multicolumn{2}{|c|}{ Median educational level ${ }^{\text {b }}$} \\
\hline 1 (17.6\% or more) & $8222(10.6 \%)$ \\
\hline 2 (10.9\%-17.5\%) & $16,236(21.0 \%)$ \\
\hline $3(6.3 \%-10.8 \%)$ & $26,636(34.5 \%)$ \\
\hline $4(<6.3 \%)$ & $26,190(33.9 \%)$ \\
\hline
\end{tabular}

As shown in Table 3, factors associated with poor OS in multivariable analysis in the entire cohort of 77,425 patients included LVI (HR 1.24, $p<0.0001$ ) and Black race (HR 1.24, $p=$ 0.01 ). LVI was prognostic in the 65,018 patients with N0 (HR 1.37, $p<0.0001)$ but not in the 12,407 patients with $\mathrm{N}+(\mathrm{HR} 1.04, p=$ $0.66)$ disease, whereas Black race was prognostic in both NO (HR $1.21, p=0.009)$ and $\mathrm{N}+(\mathrm{HR} 1.37, p=0.015)$ disease. Other factors associated with poorer OS included $\mathrm{N}+$ disease $(p<0.0001)$, larger tumor size $(p<0.0001)$, grade 3 histology $(p<0.0001)$, higher RS $(11-25$ vs $0-10, p=0.032,>26$ vs $0-10, p<0.0001)$, older age $(p<$ 
Table 2. Associations between LVI, race, and other clinicopathologic features.

\begin{tabular}{|c|c|c|c|c|c|c|c|c|}
\hline & LVI present & LVI absent & LVI unknown & $P$ value & White & Black & Other & $P$ value \\
\hline 0-20 mm & 6202 (62.9\%) & $46,843(79.0 \%)$ & 6351 (76.8\%) & & $52,458$ (77. $2 \%)$ & 4192 (72.9\%) & $2308(74.6 \%)$ & \\
\hline Node involvement & & & & $<0.0001$ & & & & 0.0253 \\
\hline Negative & 6207 (63.0\%) & $52,094(87.8 \%)$ & 6717 (81.3\%) & & $57,152(84.0 \%)$ & 4761 (82.8\%) & 2622 (84.7\%) & \\
\hline 1 & $1222(12.4 \%)$ & 17,641 (29.8\%) & 2206 (26.7\%) & & $18,964(27.9 \%)$ & 1251 (21.8\%) & 707 (22.8\%) & \\
\hline 2 & 5407 (54.9\%) & 30,511 (51.5\%) & 4270 (51.7\%) & & $35,196(51.8 \%)$ & 2973 (51.7\%) & 1712 (55.3\%) & \\
\hline 3 & $2755(28.0 \%)$ & 8067 (13.6\%) & 1255 (15.2\%) & & 10,187 (15.0\%) & 1252 (21.8\%) & $544(17.6 \%)$ & \\
\hline Unknown & 472 (4.79\%) & $3086(5.2 \%)$ & $533(6.5 \%)$ & & 3651 (5.4\%) & $274(4.8 \%)$ & 133 (4.3\%) & \\
\hline Recurrence score & & & & $<0.0001$ & & & & $<0.0001$ \\
\hline
\end{tabular}

$0.0001)$, lower median income $(p=0.0001)$, and greater number of comorbidities $(p<0.0001)$.

\section{LVI, categorical RS, and overall survival in N0 disease}

Among 65,018 patients with N0 disease, in whom LVI was prognostic, the presence of LVI was associated with worse OS in those with RS 11-25 [HR $1.31(95 \% \mathrm{Cl} 1.09,1.57)]$ and 26-100 [HR $1.58(95 \% \mathrm{Cl} 1.30,1.93)]$, but not in patients with RS 0-10 [HR 1.1 $(95 \% \mathrm{Cl} 0.77,1.53)]$, although there was no statistical interaction between LVI and RS (interaction $p=0.35$ ) (Supplemental Table 2).

\section{Chemotherapy benefit}

Receipt of chemotherapy was associated with improved OS in the entire cohort $(p=0.003)$ and in $\mathrm{N}+$ patients $(p=0.001)$, but not in N0 patients $(p=0.134)$. There was no interaction between LVI and chemotherapy benefit in the 39,402 patients with N0 disease and an RS 11-25 (interaction $p=0.46$ ) (Supplemental Table 3).

\section{Comparison of patients who did and did not undergo RS assay} Of 212,394 patients in the NCDB database eligible for genomic analysis, only 77,425 (36.5\%) had numeric RS available. We compared characteristics of this group with the 119,321 patients who did not undergo RS analysis. Receipt of RS correlated with N0 disease, grade 2 histology, and absence of comorbidities $(p<$ 0.0001 ), and was inversely associated with median educational and income levels $(p<0.0001)$. Both presence of LVI and Black race were associated with lower receipt of RS $(12.7 \%$ vs $14.2 \%$ for LVI; $7.4 \%$ vs $9.2 \%$ for Black race, $p<0.0001)$. Use of RS was associated with lower likelihood of receiving chemotherapy (26.0\% vs $34.8 \%, p<0.0001)$ (Supplemental Table 4). Among women who did not have genomic testing, LVI was seen more frequently in non-white women than in white women $(13.9 \%$ for white women, vs. $15.7 \%$ for Black women, vs. $17.1 \%$ for women of other races, $p<0.0001$ ); however, the difference in frequency of LVI between white and Black women, whereas statistically significant, was numerically small in magnitude. Multivariable analysis of OS in women who did not undergo RS assay showed that LVI was associated with poorer OS in the entire cohort $(\mathrm{HR}=$ $1.21, p<0.0001)$, and in both N0 ( $\mathrm{HR}=1.15, p=0.008)$ and $\mathrm{N}+$ patients $(H R=1.22, p<0.00001)$. Black race was associated with poorer $\mathrm{OS}$ in the entire cohort ( $\mathrm{HS}=1.14, p=0.0002)$ and in $\mathrm{N}+$ (HR $=1.26, p<0.0001)$ women only (Supplemental Table 5).

\section{DISCUSSION}

We evaluated the association between LVI, race, and the 21-gene RS in 77,425 women with ER-positive, HER2-positive EBC with 0-3 positive axillary nodes. We confirmed prior reports that LVI is associated with a worse prognosis in NO disease $\mathrm{e}^{1,3,5,6}$, and that Black race is associated with a worse prognosis ${ }^{20,23,24}$, but report for the first time no association between Black race and the presence of LVI. We also report for the first time that LVI adds prognostic information to the 21-gene RS, but not predictive information for chemotherapy benefit in those with a mid-range RS of 11-25.

Three previous studies have evaluated the correlation between LVI and the 21-gene RS in ER+ NO EBC. Mutai et al. ${ }^{16}$ and Tan et al. $^{37}$ evaluated 657 and 58 tumor samples, respectively, and reported no association between LVI and numeric RS. Mutai et al also reported that the presence of LVI was associated with poorer OS in patients with intermediate RS, but not in patients with low or high RS. In contrast, Turashvili et al. ${ }^{38}$ evaluated 2326 patients with EBC, reported an association of LVI with increasing RS, and found a trend toward greater likelihood of locoregional recurrence in patients with LVI (HR 1.81, 95\% Cl 0.96, 3.42, $p=0.06)$ after adjustment for RS.

Prior to the publication of the randomized TAILORx data ${ }^{13}$, the optimal management for patients with NO ER+ BCA and intermediate RS was unclear, and recommendations for adjuvant chemotherapy in this group of patients were often based on clinicopathologic criteria. Chen et al. ${ }^{39}$ showed that the presence of LVI was significantly associated with receipt of chemotherapy in patients with intermediate RS diagnosed before 2013. Our study, although not randomized, did not detect a chemotherapy benefit in patients with intermediate RS and LVI who received adjuvant chemotherapy. In contrast, a retrospective evaluation of patients enrolled on two International Breast Cancer Study Group trials of chemoendocrine therapy in NO EBC found that the benefit of ovarian suppression with goserelin in premenopausal patients with $\mathrm{ER}+$ disease was exclusively confined to patients with $\mathrm{LV} \mathrm{I}^{40}$. In addition, analysis of the BIG 1-98 trial showed that patients with LVI had greater than average benefit from letrozole compared with tamoxifen ${ }^{41}$. These findings are consistent with results of the SOFT/TEXT trials, indicating greater benefit from ovarian function suppression plus an aromatase inhibitor compared with tamoxifen in those at higher recurrence risk ${ }^{42}$.

Multiple factors contribute to poorer prognoses for Black breast cancer patients, including disparities in access to care ${ }^{17,18}$, later 


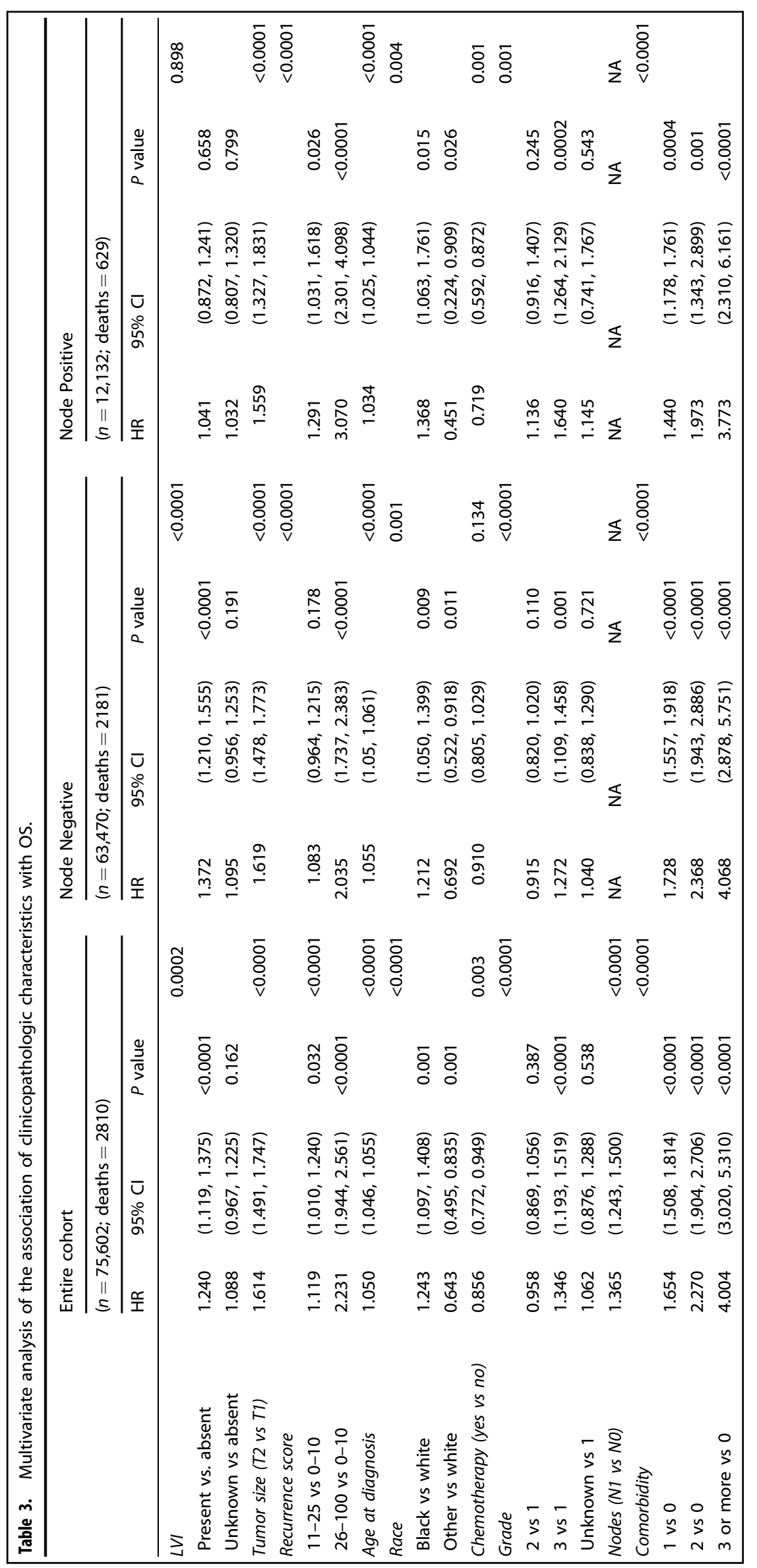


stage at diagnosis ${ }^{43,44}$, higher incidence of triple-negative disease $\mathrm{e}^{45}$, and decreased adherence to chemotherapy and endocrine therapy regimens ${ }^{46,47}$. However, four clinical trials, which collectively included 26,138 patients, 2,370 (9\%) of whom were characterized as Black (either by self-reported race, or by ancestry-informative genetic markers of African ancestry) have shown poorer outcomes for Black women with $\mathrm{HR}+\mathrm{EBC}^{20-23}$, despite similar antineoplastic therapy, indicating that differences in tumor biology in HR+ EBC also play a role. This disparity is hypothesized to be at least partially due to racial differences in the tumor microenvironment ${ }^{24,25}$. Although Black women have higher rates of obesity, which has been associated with higher recurrence rates in HR+ HER2 - disease ${ }^{48,49}$, evidence suggests that this racial disparity is not explained by obesity alone 22,50 . Our analysis, using real-world data from $\mathrm{NCDB}$, indicates that the racial disparity in outcome of ER+ EBC is not explained by a greater incidence of $\mathrm{LVI}$, or by substantially and clinically relevant differences in the 21 gene RS.

Our study has several strengths and limitations, which are inherent in the use of NCDB data. Strengths include the large sample size, utilizing real-world data from $\mathrm{CoC}$-accredited institutions, and representing the largest analysis evaluating the prognostic information provided by LVI, with a sufficient sample size to determine an association between LVI and Black race. Limitations include lack of information regarding cancer recurrence, both distant and locoregional, relatively short duration of follow-up, and relative under-representation of Black women in the EBC NCDB dataset ${ }^{43}$. An additional important limitation of our study is that the NCDB relies on local assessment of LVI, and does not distinguish between focal and extensive LVI. Prior reports indicate that up to $26 \%$ of local pathologists' assessments of LVI are altered after pathologic review by an experienced breast pathologist $^{51}$, and that extent of LVI correlates with poorer outcomes $^{6}$. In addition, $10.67 \%$ of our cohort had unknown LVI status.

Our study is also limited by real-world biases in the ordering of RS. Comparison between our cohort and patients in the NCDB database who did not undergo RS assay show that patients in whom RS was ordered were more likely to have intermediategrade tumors, N0 disease, and no comorbidities, confirming previously reported clinician tendencies to order RS in a state of clinical equipoise ${ }^{52,53}$, and in patients felt to be able to tolerate chemotherapy $^{53}$. Lower receipt of RS in patients with LVI may reflect clinician bias in favor of administering chemotherapy to patients with LVI, a known poor prognostic factor. Black women were also somewhat under-represented in our cohort, compared with patients who did not receive RS. Previous studies evaluating racial bias in receipt of genomic testing have shown conflicting results. One study utilizing NCDB data showed that Black women were less likely than white women to undergo RS assay, and more likely to have testing ordered in guideline-discordant scenarios ${ }^{54}$. However, a more recent study, utilizing data from the Georgia Cancer Registry, found no racial differences in receipt of RS, but found a 1 year lag in uptake of testing in Black women ${ }^{55}$.

In summary, our data demonstrated that LVI adds prognostic information for OS in NO ER+ HER2-BCA, with RS 11-100, but found no association between LVI and Black race in women undergoing RS assay. Despite the poorer prognosis associated with LVI, no chemotherapy benefit was seen in patient with RS 11-25 and LVI. Therefore the presence of LVI should not influence chemotherapy decision-making in patients with intermediate RS.

\section{METHODS}

\section{Case selection}

We utilized a data set derived from the 2005-2016 National Cancer Database (NCDB). NCDB is a nationwide, facility-based database jointly sponsored by the American Cancer Society and the American College of
Surgeons Commission on Cancer (CoC). The NCDB contains data collected on over 34,000,000 cancer cases from over $1500 \mathrm{CoC}$-accredited hospitals, representing over $70 \%$ of newly diagnosed cancers ${ }^{56}$, and $80 \%$ of newly diagnosed breast cancers in the United States ${ }^{57}$. The NCDB Participant User File (PUF) is a HIPAA-compliant data file, which is made available to investigators from $\mathrm{CoC}$-accredited cancer programs who complete an application process. The data used in the study are derived from a deidentified NCDB file. The American College of Surgeons and the Commission on Cancer have not verified and are not responsible for the analytic or statistical methodology employed, or the conclusions drawn from these data by the investigator. The NCDB PUF contains de-identified patient data, including demographic information, tumor site, and pathology data, first course of treatment, and mortality. The NCDB PUF does not contain information on recurrence. The NCDB began collecting information on both LVI and on results of gene expression assays in 2010.

The study population included women age 75 and under-diagnosed between 1 January 2010 and 1 January 2014 with estrogen receptorpositive (ER+) HER2 - BCA, measuring up to $5 \mathrm{~cm}$, with 0-3 pathologically involved axillary nodes, treated with definitive surgery as first treatment, and with numeric RS available. Demographic information obtained included age, race, estimated annual household income and educational attainment. Race was classified as white, Black, other (including all other racial codes captured by the NCDB) or unknown. Clinical characteristics included tumor size in $\mathrm{mm}$, histologic grade, axillary node involvement, $\mathrm{LVI}$, and numeric RS. Micrometastatic nodal involvement (pN1mi) was classified as node positive. LVI was characterized as present, absent, or unknown. RS was characterized as low, intermediate, or high using TAILORx cutpoints, where $0-10$ was defined as low, $11-25$ as intermediate, and $26-100$ as high.

\section{Statistical analysis}

Bivariate associations between categorical variables were examined using the chi-square test. Multivariate Cox proportional hazards model were used to assess the association of LVI, chemotherapy, and RS on OS, while adjusting age, race, tumor size, grade, LN status, Charlson-Deyo comorbidity score, median income, and education level. The estimated HR for each variable in the model, along with its $95 \% \mathrm{Cl}$, was reported. All tests are two-sided with a significance level $\leq 5 \%$. Proportionality assumption was examined, and no violation was detected. All analyses were conducted using SAS 9.4 (SAS Institute Inc., Cary, NC, USA).

\section{Ethics}

NCDB PUF data are de-identified, and compliant with HIPAA. Hospitals, health-care providers, and patients are not identified. Patient-informed consent is not obtained prior to institutional data submission to NCDB. As this study utilizes de-identified patient data, with no attempt made to contact or re-identify the subjects, it is deemed exempt from oversight by the Institutional Review Board of Albert Einstein College of Medicine.

\section{Reporting summary}

Further information on research design is available in the Nature Research Reporting Summary linked to this article.

\section{DATA AVAILABILITY}

The data generated and analyzed during this study are described in the following data record: https://doi.org/10.6084/m9.figshare.13517210 $0^{58}$. The data are stored in the National Cancer Database (NCDB) Participant User File (PUF) "NCDBPUF_Breast.0.2016.0.dat". This is a HIPAA-compliant data file. The data use agreement between the researchers and the NCDB prohibits sharing of data, but the file can be made available to investigators from $\mathrm{CoC}$-accredited cancer programs who complete an application process ( $\mathrm{CoC}$ is the American College of Surgeons Commission on Cancer). Information regarding the application process is available at https://www. facs.org/quality-programs/cancer/ncdb/puf.

\section{CODE AVAILABILITY}

SAS 9.4 was used, and the code is available upon request. 
Received: 15 May 2020; Accepted: 20 January 2021; Published online: 01 March 2021

\section{REFERENCES}

1. Lee, A. H. et al. Prognostic value of lymphovascular invasion in women with lymph node negative invasive breast carcinoma. Eur. J. Cancer 42, 357-362 (2006).

2. Song, Y. J. et al. The role of lymphovascular invasion as a prognostic factor in patients with lymph node-positive operable invasive breast cancer. J. Breast Cancer 14, 198-203 (2011).

3. Liao, G.-S., Hsu, H.-M., Dai, M.-S. \& Yu, J.-C. 110PPrognostic and predictive value of lymphovascular invasion and lymph node status among breast cancer subtypes. Ann. Oncol. 28, https://doi.org/10.1093/annonc/mdx363.026 (2017).

4. Ryu, Y. J., Kang, S. J., Cho, J. S., Yoon, J. H. \& Park, M. H. Lymphovascular invasion can be better than pathologic complete response to predict prognosis in breast cancer treated with neoadjuvant chemotherapy. Medicine (Baltim.) 97, e11647 (2018).

5. de Mascarel, I. et al. Obvious peritumoral emboli: an elusive prognostic factor reappraised. Multivariate analysis of 1320 node-negative breast cancers. Eur. J. Cancer 34, 58-65 (1998).

6. Colleoni, M. et al. Prognostic role of the extent of peritumoral vascular invasion in operable breast cancer. Ann. Oncol. 18, 1632-1640 (2007).

7. Voogd, A. C. et al. Differences in risk factors for local and distant recurrence after breast-conserving therapy or mastectomy for stage I and II breast cancer: pooled results of two large European randomized trials. J. Clin. Oncol. 19, 1688-1697 (2001).

8. Harris, L. N. et al. Use of biomarkers to guide decisions on adjuvant systemic therapy for women with early-stage invasive breast cancer: American Society of Clinical Oncology Clinical Practice Guideline. J. Clin. Oncol. 34, 1134-1150 (2016).

9. Krop, I. et al. Use of biomarkers to guide decisions on adjuvant systemic therapy for women with early-stage invasive breast cancer: American Society of Clinical Oncology Clinical Practice Guideline Focused Update. J. Clin. Oncol. 35, 2838-2847 (2017).

10. Paik, S. et al. A multigene assay to predict recurrence of tamoxifen-treated, nodenegative breast cancer. N. Engl. J. Med. 351, 2817-2826 (2004).

11. Paik, S. et al. Gene expression and benefit of chemotherapy in women with nodenegative, estrogen receptor-positive breast cancer. J. Clin. Oncol. 24, 3726-3734 (2006).

12. Albain, K. S. et al. Prognostic and predictive value of the 21-gene recurrence score assay in postmenopausal women with node-positive, oestrogen-receptorpositive breast cancer on chemotherapy: a retrospective analysis of a randomised trial. Lancet Oncol. 11, 55-65 (2010).

13. Sparano, J. A. et al. Adjuvant chemotherapy guided by a 21-gene expression assay in breast cancer. N. Engl. J. Med. 379, 111-121 (2018).

14. Gluz, O. et al. West German Study Group Phase III PlanB Trial: first prospective outcome data for the 21-gene recurrence score assay and concordance of prognostic markers by central and local pathology assessment. J. Clin. Oncol. 34, 2341-2349 (2016).

15. Nitz, U. et al. Reducing chemotherapy use in clinically high-risk, genomically lowrisk pN0 and pN1 early breast cancer patients: five-year data from the prospective, randomised phase 3 West German Study Group (WSG) PlanB trial. Breast Cancer Res. Treat. 165, 573-583 (2017).

16. Mutai, R. et al. Prognostic value of the detection of lymphovascular invasion in hormone receptor-positive early breast cancer in the era of molecular profiling. Oncology 96, 14-24 (2019).

17. Williams, F. \& Thompson, E. Disparities in breast cancer stage at diagnosis: importance of race, poverty, and age. J. Health disparities Res. Pract. 10, 34-45 (2017).

18. Menashe, I., Anderson, W. F., Jatoi, I. \& Rosenberg, P. S. Underlying causes of the black-white racial disparity in breast cancer mortality: a population-based analysis. J. Natl. Cancer Inst. 101, 993-1000 (2009).

19. Albain, K. S., Unger, J. M., Crowley, J. J., Coltman, C. A. Jr. \& Hershman, D. L. Racial disparities in cancer survival among randomized clinical trials patients of the Southwest Oncology Group. J. Natl. Cancer Inst. 101, 984-992 (2009).

20. Sparano, J. A. et al. Long-term follow-up of the E1199 phase III trial evaluating the role of taxane and schedule in operable breast cancer. J. Clin. Oncol. 33, 2353-2360 (2015).

21. Schneider, B. P. et al. Impact of genetic ancestry on outcomes in ECOG-ACRINE5103. JCO Precis. Oncol 2017, https://doi.org/10.1200/PO.17.00059 (2017).

22. Sparano, J. A. et al. Race and hormone receptor-positive breast cancer outcomes in a randomized chemotherapy trial. J. Natl. Cancer Inst. 104, 406-414 (2012).
23. Albain, K. S. et al. in San Antonio Breast Cancer Symposium (San Antonio, TX, 2018).

24. Deshmukh, S. K. et al. Emerging evidence for the role of differential tumor microenvironment in breast cancer racial disparity: a closer look at the surroundings. Carcinogenesis 38, 757-765 (2017).

25. Kim, G. et al. The contribution of race to breast tumor microenvironment composition and disease progression. Front. Oncol. 10, 1022 (2020).

26. Kariri, Y. A. et al. Molecular complexity of lymphovascular invasion: the role of cell migration in breast cancer as a prototype. Pathobiology 87, 218-231 (2020).

27. Klahan, S. et al. Identification of genes and pathways related to lymphovascular invasion in breast cancer patients: a bioinformatics analysis of gene expression profiles. Tumour Biol. 39, 1010428317705573 (2017).

28. Aleskandarany, M. A., Sonbul, S. N., Mukherjee, A. \& Rakha, E. A. Molecular mechanisms underlying lymphovascular invasion in invasive breast cancer. Pathobiology 82, 113-123 (2015).

29. Martin, D. N. et al. Differences in the tumor microenvironment between AfricanAmerican and European-American breast cancer patients. PLOS ONE 4, e4531 (2009).

30. Hong, C. C. et al. Genetic variants in immune-related pathways and breast cancer risk in african american women in the amber consortium. Cancer Epidemiol. Biomark. Prev. 27, 321-330 (2018).

31. Lindner, R. et al. Molecular phenotypes in triple negative breast cancer from African American patients suggest targets for therapy. PLOS ONE 8, e71915 (2013).

32. Koru-Sengul, T. et al. Breast cancers from black women exhibit higher numbers of immunosuppressive macrophages with proliferative activity and of crownlike structures associated with lower survival compared to non-black Latinas and Caucasians. Breast Cancer Res. Treat. 158, 113-126 (2016).

33. Riabov, V. et al. Role of tumor associated macrophages in tumor angiogenesis and lymphangiogenesis. Front. Physiol. 5, 75 (2014).

34. Klingen, T. A., Chen, Y., Aas, H., Wik, E. \& Akslen, L. A. Tumor-associated macrophages are strongly related to vascular invasion, non-luminal subtypes, and interval breast cancer. Hum. Pathol. 69, 72-80 (2017).

35. Schoppmann, S. F. et al. VEGF-C expressing tumor-associated macrophages in lymph node positive breast cancer: impact on lymphangiogenesis and survival. Surgery 139, 839-846 (2006).

36. Matsumoto, K. et al. Racial differences in the outcome of patients with urothelial carcinoma of the upper urinary tract: an international study. BJU Int. 108, E304-309 (2011).

37. Tan, A. C. et al. Correlating Ki67 and other prognostic markers with Oncotype DX recurrence score in early estrogen receptor-positive breast cancer. Asia-Pac. J. Clin. Oncol. 14, e161-e166 (2018).

38. Turashvili, G. et al. 21-Gene recurrence score and locoregional recurrence in lymph node-negative, estrogen receptor-positive breast cancer. Breast Cancer Res. Treat. 166, 69-76 (2017).

39. Chen, J., Wu, X., Christos, P. J., Formenti, S. \& Nagar, H. Practice patterns and outcomes for patients with node-negative hormone receptor-positive breast cancer and intermediate 21-gene recurrence Scores. Breast Cancer Res. 20, 26 (2018).

40. Viale, G. et al. Adverse prognostic value of peritumoral vascular invasion: is it abrogated by adequate endocrine adjuvant therapy? Results from two International Breast Cancer Study Group randomized trials of chemoendocrine adjuvant therapy for early breast cancer. Ann. Oncol. 21, 245-254 (2010).

41. Mauriac, L. et al. Predictors of early relapse in postmenopausal women with hormone receptor-positive breast cancer in the BIG 1-98 trial. Ann. Oncol. 18, 859-867 (2007).

42. Pagani, O. et al. Absolute improvements in freedom from distant recurrence to tailor adjuvant endocrine therapies for premenopausal women: results from TEXT and SOFT. J. Clin. Oncol. JCO1801967, https://doi.org/10.1200/JCO.18.01967 (2019).

43. Newman, L. A. et al. Use of the National Cancer Data Base to develop clinical trials accrual targets that are appropriate for minority ethnicity patients: a report from the American College of Surgeons Oncology Group (ACOSOG) Special Population Committee. Cancer 106, 188-195 (2006).

44. Joslyn, S. A. \& West, M. M. Racial differences in breast carcinoma survival. Cancer 88, 114-123 (2000).

45. Carey, L. A. et al. Race, breast cancer subtypes, and survival in the Carolina Breast Cancer Study. JAMA 295, 2492-2502 (2006).

46. Hershman, D. et al. Racial disparities in treatment and survival among women with early-stage breast cancer. J. Clin. Oncol. 23, 6639-6646 (2005).

47. Partridge, A. H., Wang, P. S., Winer, E. P. \& Avorn, J. Nonadherence to adjuvant tamoxifen therapy in women with primary breast cancer. J. Clin. Oncol. 21, 602-606 (2003). 
48. Pan, H. \& Gray, R. G., Group, o. b. o. t. E. B. C. T. C. Effect of obesity in premenopausal ER+ early breast cancer: EBCTCG data on 80,000 patients in 70 trials. J. Clin. Oncol. 32, 503-503 (2014).

49. Sparano, J. A. et al. Obesity at diagnosis is associated with inferior outcomes in hormone receptor-positive operable breast cancer. Cancer 118, 5937-5946 (2012).

50. Lu, Y. et al. Obesity and survival among black women and white women 35 to 64 years of age at diagnosis with invasive breast cancer. J. Clin. Oncol. 29, 3358-3365 (2011).

51. Kennecke, H. F. et al. Impact of routine pathology review on treatment for nodenegative breast cancer. J. Clin. Oncol. 30, 2227-2231 (2012).

52. Sparano, J. A. \& Paik, S. Development of the 21-gene assay and its application in clinical practice and clinical trials. J. Clin. Oncol. 26, 721-728 (2008).

53. Dinan, M. A., Mi, X., Reed, S. D., Lyman, G. H. \& Curtis, L. H. Association between use of the 21-gene recurrence score assay and receipt of chemotherapy among medicare beneficiaries with early-stage breast cancer, 2005-2009. JAMA Oncol. 1, 1098-1109 (2015).

54. Jasem, J. et al. 21-gene recurrence score assay as a predictor of adjuvant chemotherapy administration for early-stage breast cancer: an analysis of use, therapeutic implications, and disparity profile. J. Clin. Oncol. 34, 1995-2002 (2016).

55. Collin, L. J. et al. Oncotype DX recurrence score implications for disparities in chemotherapy and breast cancer mortality in Georgia. NPJ breast cancer 5, 32-32 (2019).

56. Raval, M. V., Bilimoria, K. Y., Stewart, A. K., Bentrem, D. J. \& Ko, C. Y. Using the NCDB for cancer care improvement: an introduction to available quality assessment tools. J. Surg. Oncol. 99, 488-490 (2009).

57. Mallin, K. et al. Incident cases captured in the national cancer database compared with those in U.S. population based central cancer Registries in 2012-2014. Ann. Surgical Oncol. 26, 1604-1612 (2019).

58. Makower, D., Lin, J., Xue, X. \& Sparano, J. A. Metadata record for the manuscript: lymphovascular invasion, race, and the 21 -gene recurrence score in early estrogen receptor-positive breast cancer: a national cancer database analysis. figshare https://doi.org/10.6084/m9.figshare.13517210 (2021).

\section{ACKNOWLEDGEMENTS}

Supported by United States Department of Health and Human Service grant P30 CA113330.

\section{AUTHOR CONTRIBUTIONS}

D.M.-conceptualization, investigation, writing - original draft. J.L.-data curation, formal analysis, writing-review and editing. X.X.-Data curation, formal analysis, writing-review and editing. J.A.S.-project administration, supervision, writingreview and editing.

\section{COMPETING INTERESTS}

The authors declare no competing interests.

\section{ADDITIONAL INFORMATION}

Supplementary information The online version contains supplementary material available at https://doi.org/10.1038/s41523-021-00231-x.

Correspondence and requests for materials should be addressed to D.M.

Reprints and permission information is available at http://www.nature.com/ reprints

Publisher's note Springer Nature remains neutral with regard to jurisdictional claims in published maps and institutional affiliations.

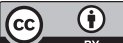

Open Access This article is licensed under a Creative Commons Attribution 4.0 International License, which permits use, sharing, adaptation, distribution and reproduction in any medium or format, as long as you give appropriate credit to the original author(s) and the source, provide a link to the Creative Commons license, and indicate if changes were made. The images or other third party material in this article are included in the article's Creative Commons license, unless indicated otherwise in a credit line to the material. If material is not included in the article's Creative Commons license and your intended use is not permitted by statutory regulation or exceeds the permitted use, you will need to obtain permission directly from the copyright holder. To view a copy of this license, visit http://creativecommons. org/licenses/by/4.0/.

(c) The Author(s) 2021 